This is an electronic reprint of the original article. This reprint may differ from the original in pagination and typographic detail.

Author(s): Erkkilä, Anna-Leena; Leppänen, Teemu; Tuovinen, Tero

Title: $\quad$ The curl and fluting of paper : The effect of elasto-plasticity

Year: $\quad 2016$

Version:

Please cite the original version:

Erkkilä, A.-L., Leppänen, T., \& Tuovinen, T. (2016). The curl and fluting of paper : The effect of elasto-plasticity. In M. Papadrakakis, V. Papadopoulos, G. Stefanou, \& V. Plevris (Eds.), ECCOMAS Congress 2016 : VII European Congress on Computational Methods in Applied Sciences and Engineering : Proceedings, Volume III (pp. 47524769). National Technical University of Athens; ECCOMAS. https://doi.org/10.7712/100016.2146.8365

All material supplied via JYX is protected by copyright and other intellectual property rights, and duplication or sale of all or part of any of the repository collections is not permitted, except that material may be duplicated by you for your research use or educational purposes in electronic or print form. You must obtain permission for any other use. Electronic or print copies may not be offered, whether for sale or otherwise to anyone who is not an authorised user. 


\title{
THE CURL AND FLUTING OF PAPER: THE EFFECT OF ELASTO-PLASTICITY
}

\author{
Anna-Leena Erkkilä ${ }^{1}$, Teemu Leppänen ${ }^{2}$ and Tero Tuovinen ${ }^{1}$ \\ ${ }^{1}$ University of Jyväskylä \\ PO Box 35, FI-40014 Jyväskylä, Finland \\ \{anna-leena.erkkila, tero.tuovinen\}@jyu.fi \\ ${ }^{2}$ LUT Savo Sustainable Technologies, Lappeenranta University of Technology, Varkaus unit \\ Opiskelijankatu 3, FI-78210 Varkaus, Finland \\ teemu.leppanen@lut.fi
}

Keywords: Elasto-plasticity, Hygroexpansivity, Paper, Finite Element Method, Curl, Fluting.

\begin{abstract}
An in-plane elasto-plastic material model and a hygroexpansivity model were applied for paper. The input parameters for both models are fiber orientation and dry solids content. A finite element model was constructed offering possibilities for studying different structural variations of an orthotropic sheet as well as buckling behavior and internal stress situations under through-thickness strain differences. Examples related to the curl and webfluting phenomena of paper are presented. Both studied cases presented in this paper indicates the usefulness of the hygro-elasto-plastic model in predicting the challenging deformation phenomena of orthotropic paper sheets. The application possibilities of the hygro-elasto-plastic model are diverse, including the investigation into several phenomena and defects appearing in drying, converting and printing process conditions.
\end{abstract}




\section{INTRODUCTION}

There are several undesired phenomena that can be considered to be connected to hygroscopic and stress-strain behavior of the paper web or sheet during production processes and in end-use. Examples of such phenomena are the development of shrinkage profiles, loose edges of web, baggy paper web causing possible wrinkling and misregistration in printing, out-ofplane deformations such as cockling, curl and fluting in heatset-web-offset (HSWO) printing process or distortion of shape of product such as sheet or box (see Fig. 1). Natural fibers and their treatments, bonds between fibers and their orientation in the fiber network, additives and manufacturing conditions all affect the sorption based dimensional instability and the mechanical properties of paper or board $[1,2,3,4,5,6,7,8,9,10]$.

Several models to predict in-plane mechanical and rheological properties, shrinkage and hygroexpansivity of paper and board have been introduced. Nonlinear elastic models, orthotropic elasto-plastic approaches and viscoelastic models have been used for studying bending, buckling, failure, tensile response, and creep and relaxation behavior of paper sheet, see, for example $[11,12,13,14,15,16]$ and $[17,18,19,20,21,22,23,24]$. The formula for the hygroexpansion of paper has been derived from the hygroexpansion of a single fiber and the efficiency of the stress transfer between fibers in [25]. In the reference [26], the traditional theory for linear thermoelasticity was applied to estimate hygroexpansion strains. An orthotropic hypoelastic constitutive model has been proposed for studies of phenomena behind the shrinkage profile [27]. In that model, the total strain including the hygroscopic strain component and the elastic modulus dependent on the moisture ratio are described by an exponential relation. The relation between strain history and tensile stiffness was assumed to be linear, and the model and isotropic inputs were calibrated by laboratory scale experiments. In the reference [28], the measured moisture dependency of material constants was utilized in the nonlinear elastic model to investigate the effect of moisture on mechanical behavior. Some studies on the buckling of paper have been introduced in $[12,29,30]$. Low tension streaks, having longer dimension, appear as buckling to baggy embodiment and may cause runnability problems [31,32]. Plastic strains caused by moisture streaks or some mechanical unevenness result in uneven tension profiles of the web. Buckling streakiness i.e. bagginess of paper web has been simulated using hygro-elasto-plastic model (LE-model) introduced in [33]. In the LE-model, the in-plane elasto-plastic constitutive material model and hygroexpansivity model as a function of dry solids content (DSC) and the anisotropy index of fiber orientation are applied for numerical solutions of the finite element method. The use of the anisotropy index parameter instead of the traditional fiber orientation anisotropy simplifies the handling of different directions in the case of anisotropic sheet and, for example, the determination of Hill's yield surface for the finite element approach is straightforward.

In this study, the LE-model is utilized to simulate results connected to two out-of-plane deformations: curl and fluting. Paper or board curl is the tendency of a flat paper or board sheet to distort and form curved surfaces of cylindrical shape (see Fig. 1). Although the basic cause of curl is simply a deformation variation of paper in its thickness direction, the processes causing the different deformations in different layers are complex and variable [34]. In the references [35] and [34], an analytical equation for sheet curl resulting from the hygroexpansion coefficients and elastic properties of the layers using elastic lamination theory has been derived. In the studies [36], [37] and [38], the hygroscopic out-of-plane deformations using the elastic constitutive model have been presented, while the elasto-plastic model has been applied in [39]. Twisted or diagonal curl modes are usually considered very harmful; an analytical equation is 

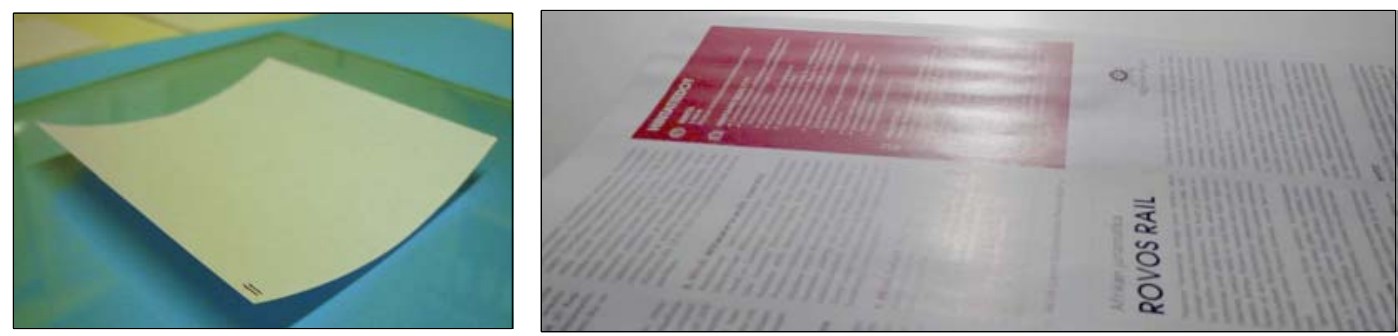

Figure 1: Examples of out-of-plane deformations of paper: picture of fine paper curl (left) and fluting in a printed area of a HSWO printed product (right).

given by Uesaka, see, for example [40]. In this paper, the LE-model is utilized to study the effect of through-thickness variation of fiber orientation and dry solids content on curl amplitude and mode.

Fluting appears as permanent, regularly spaced waves arising during heatset-web-offset printing process in the running direction of the printing machine and the machine direction (MD) of paper, see Fig. 1. Generally, two stages can be distinguished in the fluting phenomenon: fluting formation and fluting retention. There is, however, no final conclusion of the mechanisms of these two stages. For fluting formation, at least three more or less different explanations have been offered. A phenomenon related to tension wrinkles [41, 42] and differential shrinkage of inked and non-inked areas due the moisture difference $[43,44]$ are two of the proposed reasons. The occurrence of moisture difference is also confirmed by measurements $[45,46]$. Thirdly, small local in-plane moisture content variations have been linked to the formation of fluting [47]. It has been suggested that the ink layer has an effect on the permanency of fluting $[41,43]$. However, in [47] it has been estimated that ink stiffening alone cannot explain the amplitudes observed in practice. Based on simulations and experimental study, he suggests that irreversible shrinkage and thermal plastic deformations of paper may be additional mechanisms for fluting to achieve a required retention. Earlier simulation studies concerning the fluting phenomenon are based on elastic material models, see for example [44, 38, 47]. However, when fluting retention and trough-thickness moisture gradients are concerned, an elasto-plastic modeling approach could be very convenient, as simulation results of the LE-model presented in this paper indicates.

\section{MEASUREMENTS}

\subsection{Fiber orientation anisotropy}

The fiber orientation parameters are measured using the layered fiber orientation method developed by Erkkilä [48, 49, 50]. Adhesive or lamination tape splitting was applied to paper sheet to separate approximately 10 individual layers from a paper sheet. Then the sample layers were placed against a black background, and an image of a suitable area was captured by scanner using reflective illumination. In analyzing the layer images, the aim is to detect the edges of fibers or fiber bundles and to determine their orientations. The detection of edges is based on the computation of image gradients in every image element. For a discrete digital image, the derivatives $\frac{\partial f}{\partial x}$ and $\frac{\partial f}{\partial y}$ can be approximated through a discrete differentiation operator. The operator uses two kernels $k_{x}(i, j)$ and $k_{y}(i, j)$ that are convolved with the original image $f(x, y)$ to calculate approximations of the horizontal and vertical derivatives by 


$$
\begin{aligned}
& \frac{\partial f(x, y)}{\partial x} \approx D_{x}(x, y)=\left(f * k_{x}\right)(x, y), \\
& \frac{\partial f(x, y)}{\partial y} \approx D_{y}(x, y)=\left(f * k_{y}\right)(x, y),
\end{aligned}
$$

where $*$ denotes the 2-dimensional convolution operation. The coefficients of the kernels used are based on the principle of binomial filter design [50]. The magnitude (length) $|\nabla f(x, y)|$ and direction $\theta_{f}(x, y)$ of gradient vectors at each local image point are calculated by applying Eqs. (3) and (4)

$$
\begin{gathered}
|\nabla f(x, y)|=\sqrt{D_{x}^{2}+D_{y}^{2}}, \\
\theta_{f}(x, y)=\tan ^{-1} \frac{D_{y}}{D_{x}} .
\end{gathered}
$$

The magnitude of gradient vector is directly related to the probability that the part of the image examined represents the edge of the fiber. At the fiber edge, the direction of the gradient corresponds to the direction normal to that segment of fiber edge. The discrete orientation distribution is then formed as a weighted probability density function of the local orientations $P\left(\theta_{P}\right)$, where the weighting factor is the gradient magnitude $|\nabla f(x, y)|$. The direction histogram is

$$
P\left(\theta_{P}\right)=\frac{\Sigma_{x, y}\left(|\nabla f(x, y)| \delta_{\theta_{f(x, y)}, \theta_{P}}\right)}{\Sigma_{x, y}|\nabla f(x, y)|}
$$

where $\delta_{\theta_{f(x, y)}, \theta_{P}}$ is Kronecker's delta function and $\left\{\theta_{P}, \theta_{f} \in Z: 0 \leq \theta_{P}, \theta_{f}<360\right\}$. The main direction of the orientation (orientation angle) $\theta$ is defined as the deviation of the longer symmetry axis from the machine direction. The anisotropy of the fiber orientation distribution $\xi$ is defined as a ratio of the maximum distribution value $P(\theta)$ and the value in the perpendicular direction to the maximum value $P(\theta+90)$. Examples of the layer image, the gradient magnitude, and the direction histogram are presented in Fig. 2.

\subsection{Hygroexpansion coefficient measurements}

A mixture of two pulps containing $71 \%$ thermomechanical pulp and $29 \%$ bleached softwood draft pulp was used in this study. The oriented laboratory sheets were formed using a dynamic sheet former with the target basis weight $65 \mathrm{~g} / \mathrm{m}^{2}$. The sheets were wet-pressed according to ISO5269-1:2005, and then were either air-dried freely or under restraint in standard atmosphere conditions ( 23 Celsius, $50 \% \mathrm{RH}$ ). The procedure for the hygroexpansivity measurements followed the general guidelines stated in ISO 8226-1:1994 for maximum relative humidity up to $68 \%$. According to ISO 8226-1:1994, the hygroexpansive strain $X$ is

$$
X=\frac{l_{66}-l_{33}}{l_{50}} 100 \%
$$

where $l$ is the length in the specific $\mathrm{RH} \%$ indicated by the subscript. However, the hygroexpansion coefficient $\beta(\% / \%)$, defined as

$$
\beta=\frac{X}{\Delta M}
$$

where $\Delta M(\%)$ is the moisture content change, was used in this study. At least seven parallel samples were measured in each anisotropy level. The same image analysis based method 

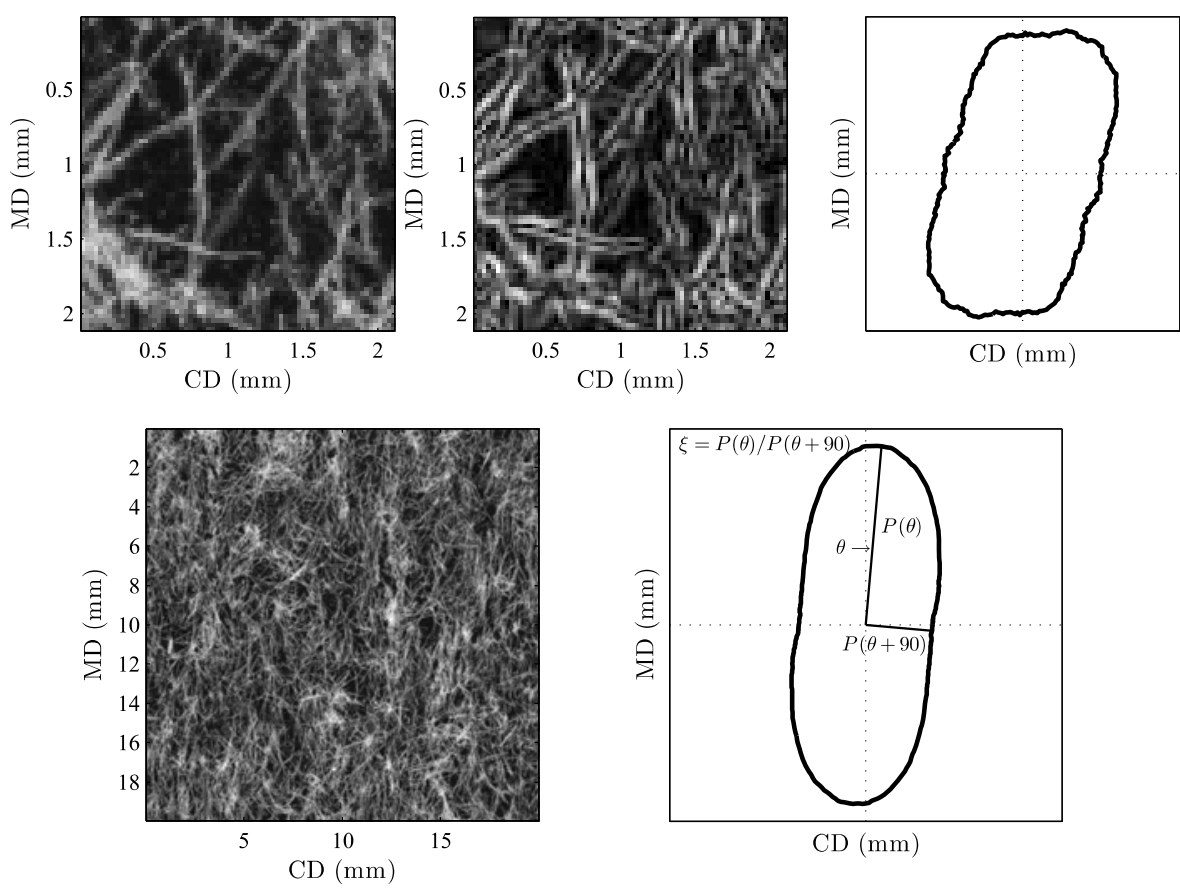

Figure 2: A $2.1 \mathrm{~mm} \times 2.1 \mathrm{~mm}$ region of newsprint layer image (top left), corresponding magnitude of gradient (top middle) and orientation distribution i.e. direction histogram (top right). A $20 \mathrm{~mm} \times 20 \mathrm{~mm}$ region of layer image (bottom left) and corresponding direction histogram, direction $1(\mathrm{P}(\theta))$, direction $2(\mathrm{P}(\theta+90))$, orientation angle $\theta$ and anisotropy $\xi$ (bottom right).

was also applied to determine the drying shrinkage of freely dried samples as a percent relative in-plane dimensional change (\%) during drying. Sheet forming and measurements of hygroexpansivity as well as drying shrinkage are described in detail in [51].

\subsection{Stress-strain tests}

The fine paper samples with three different fiber orientation anisotropy levels were produced by pilot machine. The measured averaged anisotropy values of the studied samples was 1.20 , 1.51 and 2.02. The dry solids contents varied from $53 \%$ to $56 \%$ at the initial stage. The basis weight of samples was from $77 \mathrm{~g} / \mathrm{m}^{2}$ to $80 \mathrm{~g} / \mathrm{m}^{2}$. The stress-strain curves were measured from paper strips (width $20 \mathrm{~mm}$ ) with a span length of $180 \mathrm{~mm}$. Strips were cut from the long side along four different in-plane directions $\gamma: 0,45,70$ and 90 degrees in relation to cross direction (CD) perpendicular to MD. The samples were dried in a tensile test machine to five target DSC levels: $55 \%, 65 \%, 75 \%, 85 \%$ and $95 \%$. However, the measured DSC values during each measurement were read and used in studies instead of the target DSC values. Drying shrinkage was prevented in the loading direction. The tensile stress caused by restrained shrinkage was, however, released by unloading a tensile test specimen before the load-elongation measurement was started. Three repetitions of measurements on each anisotropy, cutting direction and dry solids content combinations were performed. A description of the measuring method is presented in reference [39]. In this study, the parallel results were used as individual test points so that the total amount of data in the fitting procedure was 180 individual stress-strain curves. 
Table 1: The fitting parameters of Eq. (10) for the yield stress $\sigma_{y}$, the yield strain $\varepsilon_{y}$ and the hardening constant $H$ [33].

\begin{tabular}{l|llll}
\hline & $A_{1}$ & $A_{2}$ & $A_{3}$ & $n$ \\
\hline$\sigma_{y}$ & $-5.9030\left(\mathrm{~Pa}^{n}\right)$ & $3.1959\left(\mathrm{~Pa}^{n}\right)$ & $18.3077\left(\mathrm{~Pa}^{n}\right)$ & $0.1760(-)$ \\
$\varepsilon_{y}$ & $380.4181(-)$ & $14.3408(-)$ & $-269.8327(-)$ & $-0.7720(-)$ \\
$H$ & $-0.6021\left(\mathrm{~Pa}^{2 n}\right)$ & $4.0423\left(\mathrm{~Pa}^{2 n}\right)$ & $11.3795\left(\mathrm{~Pa}^{2 n}\right)$ & $0.0715(-)$
\end{tabular}

\section{MODELS FOR MATERIAL BEHAVIOR}

Material behavior under external and internal tensions and dry solids content changes are described by elasto-plastic material model and the hygroexpansivity model. Elasto-plastic material model is based on the curve fittings presented in [52] where equation

$$
\sigma=\left\{\begin{array}{lll}
E \varepsilon & \text { if } & \varepsilon \leq \varepsilon_{y} \\
E \varepsilon_{y}-\frac{H}{2 E}+\sqrt{H\left(\frac{H}{4 E^{2}}+\varepsilon-\varepsilon_{y}\right)} & \text { if } & \varepsilon>\varepsilon_{y}
\end{array}\right.
$$

was found suitable for describing all the uniaxial stress-strain relationships considered. In Eq. (8), $\sigma$ and $\varepsilon$ are the stress and strain, respectively; the dry solids content $R_{s c}=[0,1]$ and the anisotropy index $\phi$ dependent fitting parameters are the elastic modulus $E$, the yield strain $\varepsilon_{y}$ and the hardening constant $H$. The anisotropy index $\phi$ is defined as [52]

$$
\phi=\sqrt{\frac{1-\xi^{2}}{\xi+\tan ^{2} \gamma / \xi}+\xi}
$$

where $\xi$ is the fiber orientation anisotropy and $\gamma$ is the angle from the minor axis of the fiber orientation distribution [52]. The material model was constructed by fitting the equation [33]

$$
P=\left(A_{1}+A_{2} \phi+A_{3} R_{s c}\right)^{1 / n} \quad P=\left\{\sigma_{y}, \varepsilon_{y}, H\right\}
$$

for the dry solids content and the anisotropy index dependence. In Eq. (10), the parameters $A_{1}$, $A_{2}, A_{3}$ and $n$ are the fitting constants, see Table 1 .

The hygroexpansivity model is based on the measurements and fitting procedures presented in [53] and [33]. The hygroexpansion coefficients $\beta$ for every in-plane direction are defined by the equation

$$
\beta=\frac{k \phi^{v}}{R_{s c}^{2}} \exp \left(\frac{k \phi^{v}}{-\frac{1}{a} k \phi^{v}+\frac{b}{a}\left(1-\exp \left(-100 \frac{k \phi^{v}}{a}\right)\right)}\left(\frac{1}{R_{s c}}-1\right)\right)
$$

where the fitting parameters have values $k=0.0439, v=-0.9015, a=2.5054$ and $b=0.0250$ [33].

In the continuum mechanical model, plane stress is assumed and the yield surface is described by the Hill's yield function [54]. When the Hoffman's approximation is used as in [39] the yield surface has the form

$$
f(\sigma)=\sqrt{\sigma_{1}^{2}-\sigma_{1} \sigma_{2}+\left(\frac{\sigma_{y, 1}}{\sigma_{y, 2}}\right)^{2}\left(\sigma_{2}^{2}-\sigma_{12}^{2}\right)+\left(\frac{2 \sigma_{y, 1}}{\sigma_{y, 45^{\circ}}}\right)^{2} \sigma_{12}^{2}}
$$



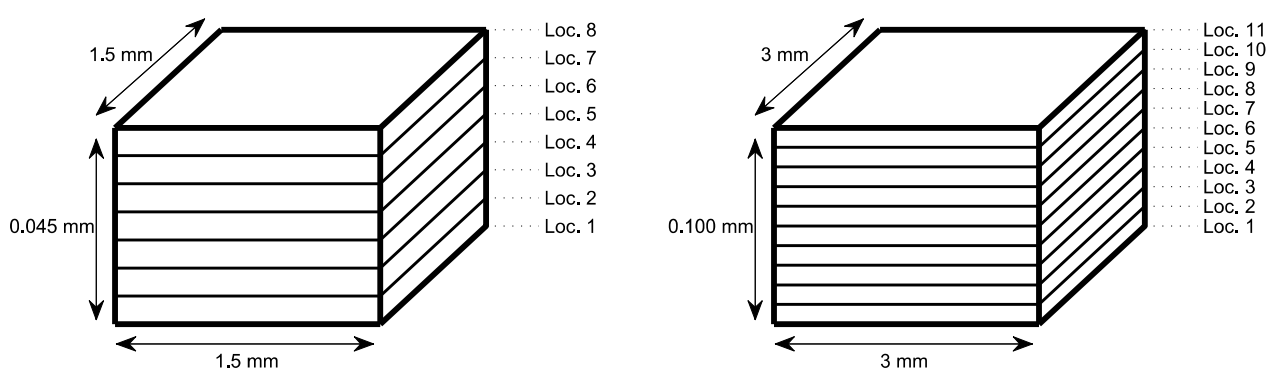

Figure 3: Elements used for the fluting (left) and the curl (right) simulations. Thickness directional DSC profile is defined by the locations shown.

where $\sigma_{1}, \sigma_{2}$ and $\sigma_{12}$ are the stress tensor components and $\sigma_{y, 1}, \sigma_{y, 2}$ and $\sigma_{y, 45^{\circ}}$ are the yield stresses in the main direction, cross direction and the direction deviating 45 degrees from the main direction, respectively. The elastic modulus is defined for directions 1,2 and $45^{\circ}$ by the yield stresses $\sigma_{y, i}$ and the yield strains $\varepsilon_{y, i}$ as

$$
E_{i}=\frac{\sigma_{y, i}}{\varepsilon_{y, i}}
$$

The direction is specified by the subscript $i$. The in-plane yield stress and yield strain parameters are defined by Eq. (10) and the shear modulus is approximated by the equation [55]

$$
G_{12}=\frac{1}{\left(\frac{4}{E_{45^{\circ}}}-\frac{1}{E_{1}}-\frac{1}{E_{2}}-\frac{2 \nu_{12}}{E_{1}}\right)}
$$

where $\nu_{12}$ is the Poisson ratio defined as

$$
\nu_{12}=\left(0.015\left(1-R_{s c}\right)+0.15\right) \phi_{1}
$$

In Eq. (15), $\phi_{1}$ is the anisotropy index of the main direction. The dependence of $\nu_{12}$ on the anisotropy index and dry solids content is roughly based on the results presented in [28] and by Maxwell relation the Poisson ratio $\nu_{21}$ is defined as $\nu_{21}=\nu_{12} E_{2} / E_{1}$.

\section{NUMERICAL SOLUTION AND SAMPLE SETUP}

Finite element method (FEM) was used to obtain the numerical solutions. Simulations were performed by the commercial software ABAQUS/Standard using shell element S4R with composite structure, see [56]. Two different phenomena were considered: fluting and curl of paper. The element size and thickness of the simulated sample are given in Fig. 3. In both cases, one element is used in the thickness direction of the sample. In the fluting simulations, the element was divided in the thickness direction into 7 layers with equal thicknesses, whereas in the curl simulations, the element was divided into 10 layers. The in-plane layouts used in the simulations are shown in Fig. 4. Automatic incrementation control and volume-proportional damping were utilized to obtain the numerical solutions [56]. For the fluting and curl simulations the damping factors $10^{-8}$ and $10^{-12}$ were used, respectively. 


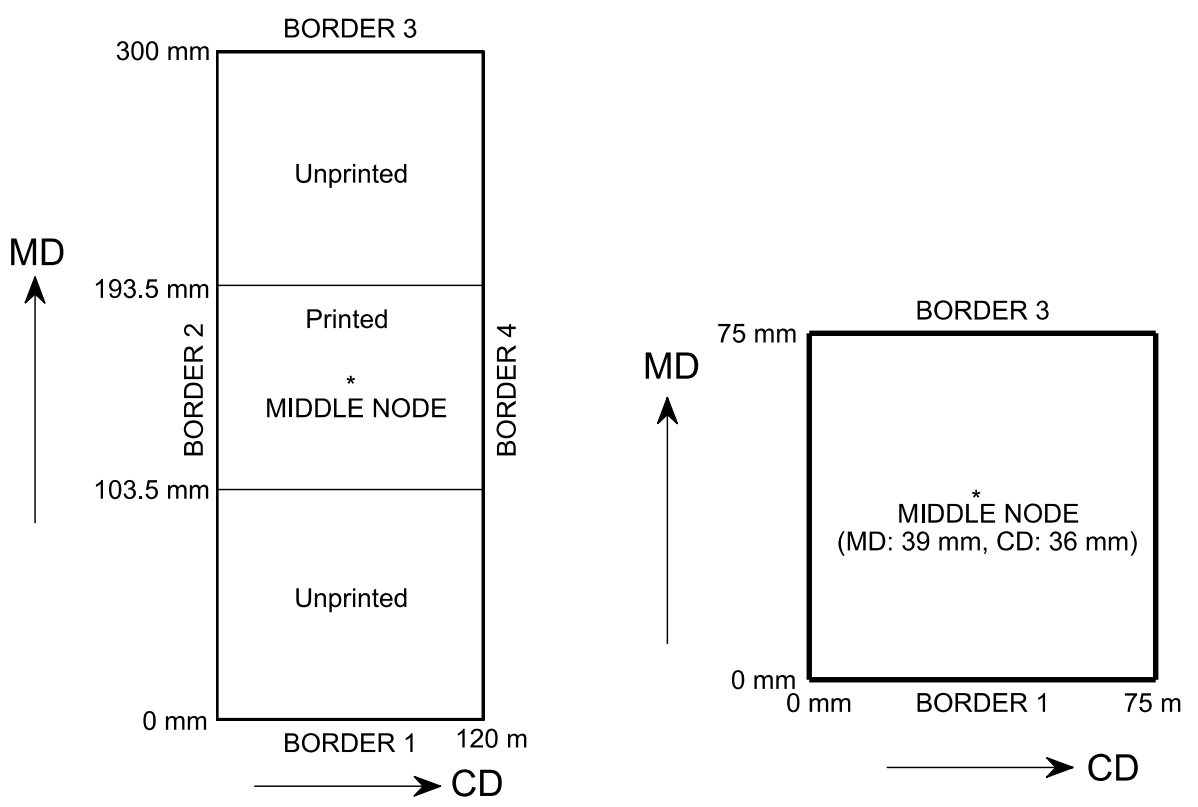

Figure 4: Layouts for fluting (left) and curl (right) simulations.

\subsection{Curl simulations}

The through-thickness variation of the fiber orientation of the samples is presented in Fig. 5. The initial DSC was $85 \%$ throughout the sample. In step 1, MD tension $200 \mathrm{~N} / \mathrm{m}$ was applied via borders 1 and 3 and the following boundary conditions were applied:

- Boundary condition 1: Out-of-plane displacement $\left(u_{3}\right)$ was restrained in all nodes.

- Boundary condition 2: In the middle node (not located into geometrical middle point, see Fig. 4) all displacements and rotations were restrained.

In step 2, the DSC profile were applied to the sample. Different DSC profiles used in step 2 are presented in Fig. 6. In step 3, the whole sample was dried into DSC 95\%. In step 4, the external tension was removed and in step 5, the boundary condition 1 was removed.

\subsection{Fluting simulations}

In the fluting simulations, a homogeneous strongly MD-orientated sample $(\theta=0, \xi=4)$ was used. The initial DSC was $96 \%$ throughout the sample. In the following, the displacements and the rotations are marked as $u_{1}$ (coincides with the MD when $\theta=0$ ), $u_{2}$ (coincides with the $\mathrm{CD}$ when $\theta=0), u_{3}$ and $u r_{1}, u r_{2}, u r_{3}$, respectively, see [56]. The boundary conditions were defined as (see Fig. 4):

- Out-of-plane displacement $\left(u_{3}\right)$ was restrained in the borders 1, 2, 3 and 4.

- The rotation $u r_{1}$ was restrained in the borders 2 and 4 .

- The rotation $u r_{2}$ was restrained in the borders 1 and 3 .

- In the middle node the displacements $u_{1}$ and $u_{2}$ and rotation $u r_{3}$ were restrained. 

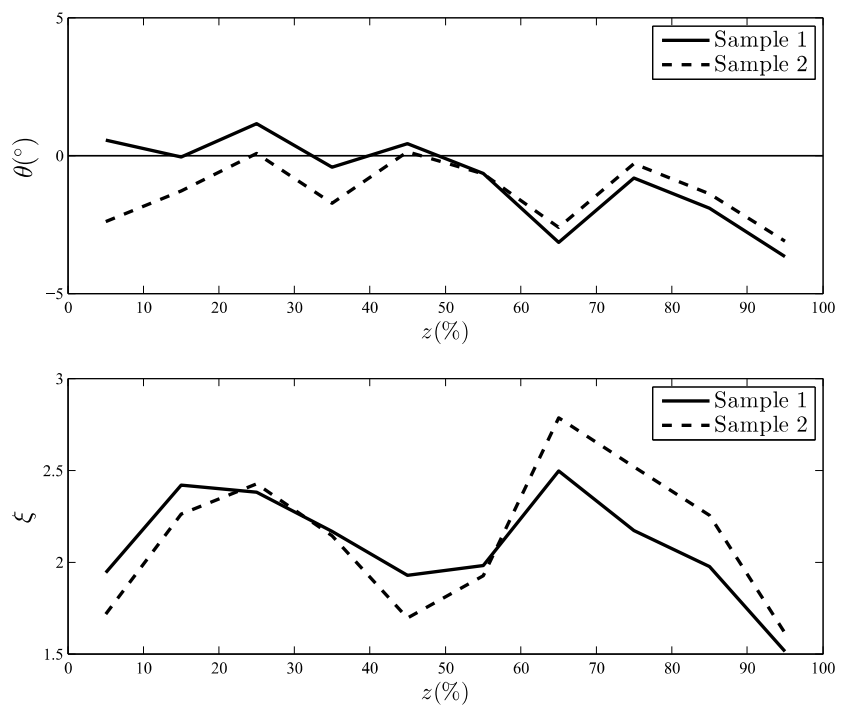

Figure 5: Through-thickness $(z)$ variation of the orientation angle $\theta$ (top) and the anisotropy $\xi$ (bottom) of the samples used in the curl simulations.

In step 1, the DSC profile (see Fig. 7) was applied to the printed area and the through-thickness symmetry was nullified by setting the out-of-plane directional force with value $10^{-3} \mathrm{~N}$ to the node located at position $\mathrm{MD}=190.5 \mathrm{~mm}, \mathrm{CD}=42 \mathrm{~mm}$. In step 2, this force was removed. In step 3, MD tension $400 \mathrm{~N} / \mathrm{m}$ was applied via borders 1 and 3 and in step 4, the DSC profile applied to the printed area in step 1 was replaced by the initial dry solids content $96 \%$. The simulations were ended by step 5 where the external tension was removed from borders 1 and 3.

\section{RESULTS AND DISCUSSION}

Curl may have different modes: curl toward surface 1 or surface 2 and cylindrical shape main axis along MD (MD curl), along CD (CD curl) or to some other angle (diagonal curl).
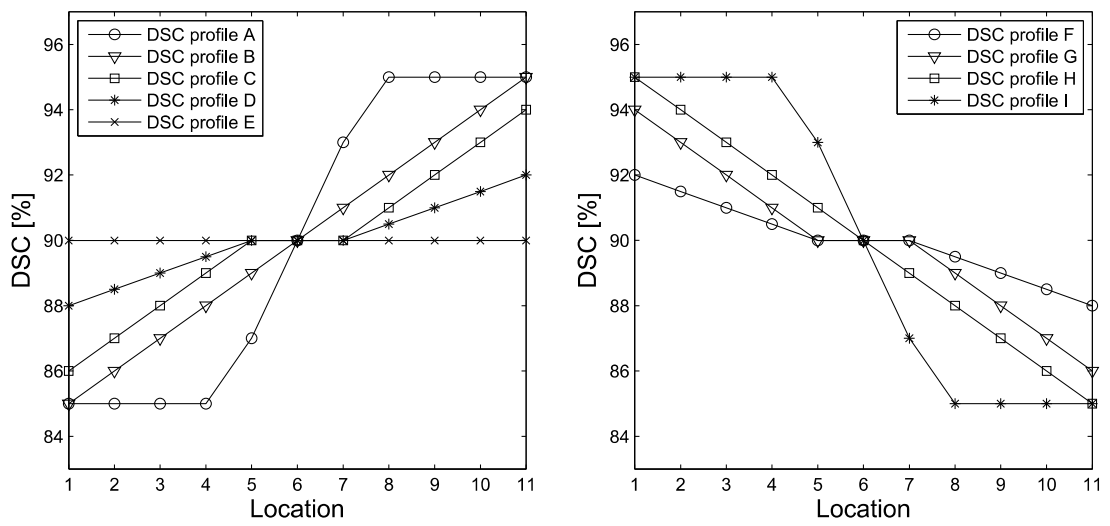

Figure 6: Curl simulations: DSC profiles. 


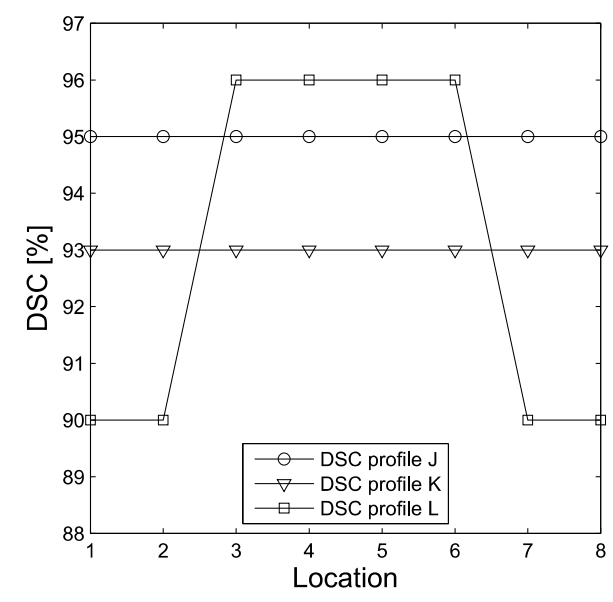

Figure 7: Fluting simulations: DSC profiles.

The simulated curl of samples 1 and 2 with all DSC profiles A-I is presented in Figs. 8 and 9. With flat drying profile $\mathrm{E}$, the curl amplitude of both samples is minor, because of fairy symmetrical anisotropy profile between sides of the samples. When drying includes profiles D or F no significant change in curl can be detected. This indicates that no plastic strains either at surface 1 or 2 have been induced. However, higher gradient in DSC profiles (A-C and G-I) generates plastic strains that change the curl. Generally, paper curls toward the surface dried last [9], i.e. dryings using DSC profiles A, B and C turn curl more toward surface 1 and DSC profiles G, H, I toward surface 2. The equivalency of strain at both sides of samples can be adjusted by drying two-sidedness. However, near the equivalent strain condition, the sample is at risk to manifest diagonal or CD mode of curl. Diagonal and CD curl are harmful modes of curl in many applications and there have been attempts to control this by adjusting the two-sidedness of orientation angle. Obviously, zero orientation angles at both sides of samples would provide the best results, but even when there is only minor orientation angle two-sidedness, as in sample 2 , there is no guarantee of maintaining MD curl mode at every drying profile. The clearest example of this phenomenon can be observed in tests carried out in single-sided and doublesided printing by either side toward heated roller of the copier or printer, see, for example [57].

Out-of-plane deformations of steps 3, 4 and 5 of fluting simulation with DSC profile L are presented in Fig. 10. Corresponding plastic deformations in MD and CD at step 5 are presented in Fig. 11. Due to the higher moisture content the printed area expands according to the hygroexpansion tendency. The tension and dryer unprinted areas constrain the printed area in CD and force it to buckle to a wavy form. The strong waviness at the printed area in step 3 can generate plastic strain especially to concave positions of waves. The leveling of the moisture difference in step 4 does not remove the plastic deformations formed already and permanent wavy deformations are produced to the sample. A higher moisture difference between printed and unprinted areas and unevenness of the moisture content profile increase severity of fluting waviness, see Fig. 12. The wavelength of simulated fluting is around $20 \mathrm{~mm}$ (see Fig. 12), which fit into the range of typically observed fluting wavelength approximately between 5 and $30 \mathrm{~mm}$, see, for example [58]. However, the wavelength is dependent on boundary conditions, sample and element size, disturbances, etc. 

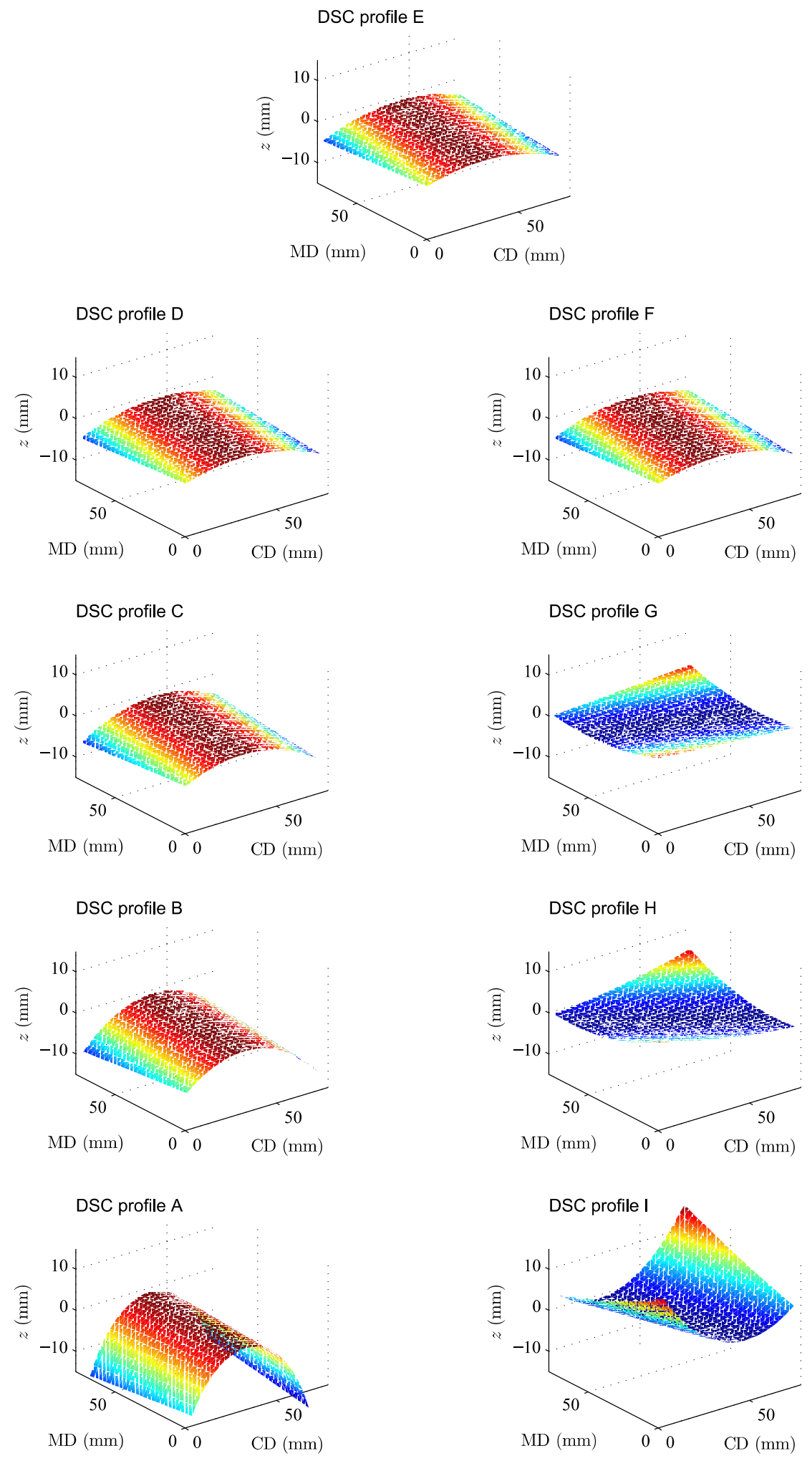

Figure 8: Simulated curl of sample 1. 

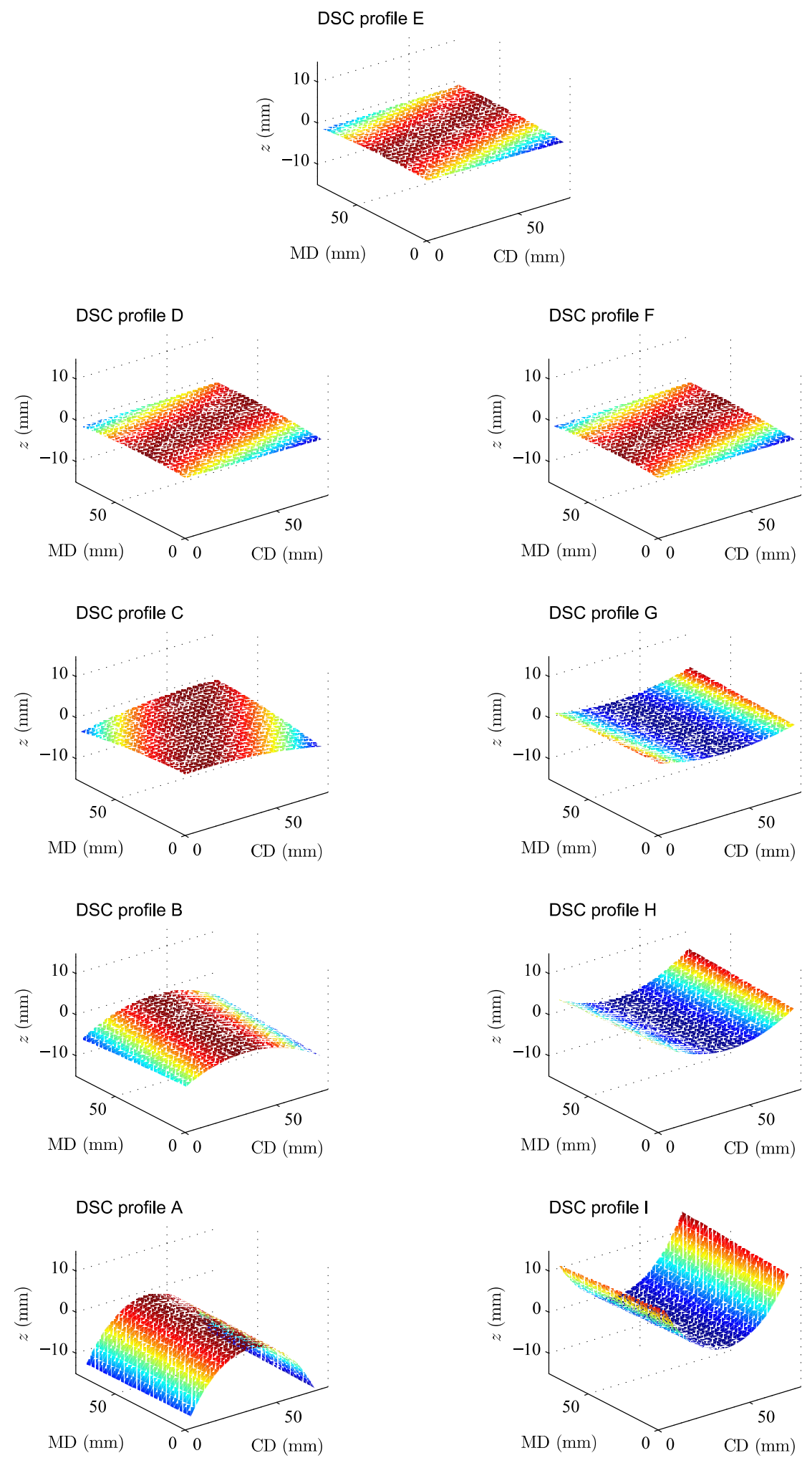

Figure 9: Simulated curl of sample 2. 

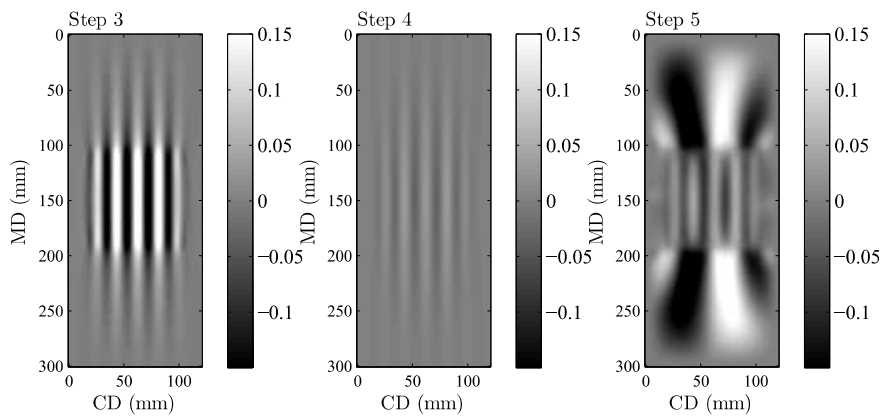

Figure 10: Simulated fluting with DSC profile L. In step 3 (left) both the external tension and the DSC profile are valid. In step 4 (middle) the DSC profile is removed and in step 5 (right) also the external tension is nullified.
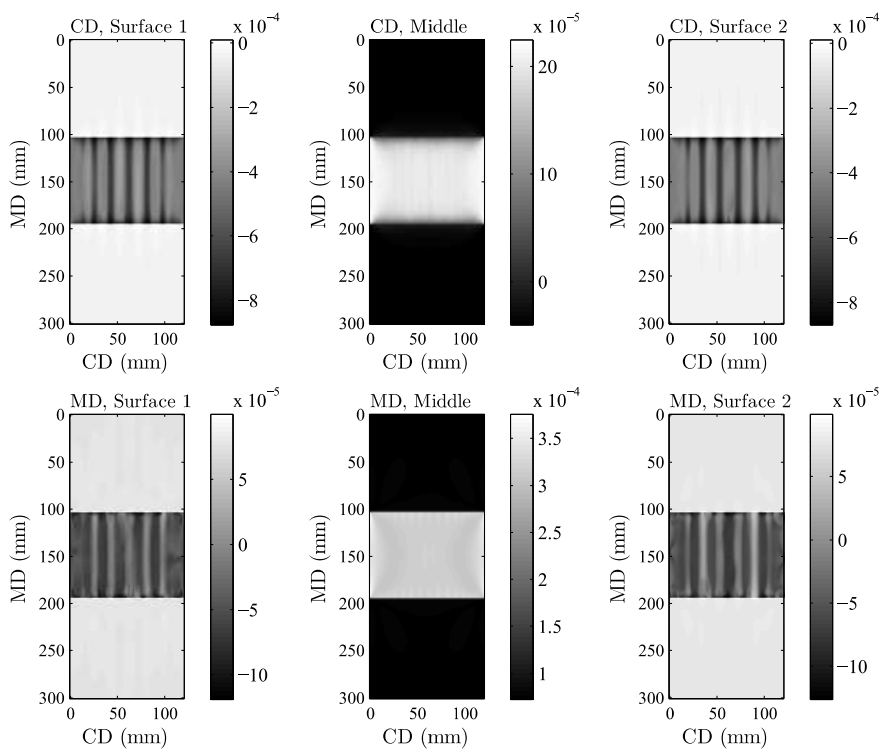

Figure 11: Plastic CD (top) and MD (bottom) strains for the layers: Surface 1 (left), Middle (middle) and Surface 2 (right). Simulations were performed with DSC profile L.

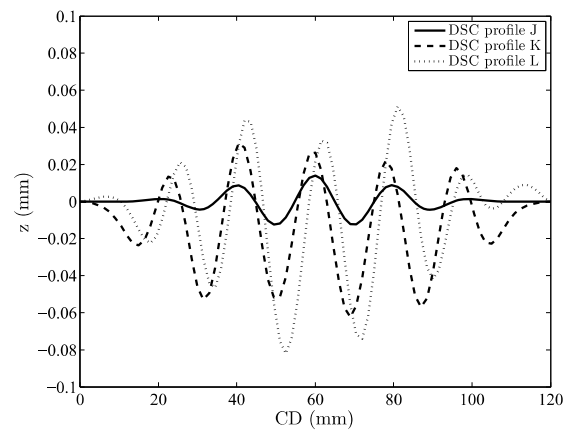

Figure 12: Cross-sections of the simulated fluting from the middle $(\mathrm{MD}=150 \mathrm{~mm})$ of the sample. 


\section{CONCLUSIONS}

There are several undesired phenomena that can be considered to be connected to hygroscopic and stress-strain behavior of paper web or sheet during production processes and in end-use. Out-of-plane deformation defects such as curl and fluting in heatset-web-offset printing have been studied widely by different experimental and modeling approaches, but there are still features left unknown.

The objective of this work was to apply a hygro-elasto-plastic model to the out-of-plane deformation phenomenon corresponding to copy curl and HSWO fluting conditions. In both cases, the simulations predict the behavior of hygroscopic orthotropic sheet under moisture change during different external in-plane stretch or stress conditions. The model includes two experimental models: elasto-plastic material model and hygroexpansivity model. In both models, the structural properties of the sheet are described by fiber orientation probability distribution, and both models are functions of the dry solids content and fiber orientation anisotropy index. The anisotropy index simplifies the procedure of determining the constitutive parameters of the material model and hygroexpansion coefficients in different directions of an anisotropic sheet. For studies of the combined and more complicated effects of hygro-elasto-plastic behavior, these two models were implemented in a finite element program for numerical solution.

The behavior of two orientated structures was simulated using different $z$-directional DSC profiles. The plastic strains were generated at least $8 \%$ and higher differences between surfaces in DSC profiles. It has been noticed that DSC differences between surfaces of paper can reach over $10 \%$ in a paper machine drying $[59,60]$. The equivalency of strain at both sides of samples can be adjusted by drying two-sidedness. This is a practically utilized method to control curl amplitude, but has not been predicted by simulation before. Near the equivalent strain condition, however, the sample is at risk to manifest diagonal or CD mode of curl. Results also predict phenomena that are commonly observed in copier printing tests.

Although the generation of HSWO fluting have been successfully predicted by modeling, the mechanisms behind permanency of HSWO fluting has been a demanding task to solve. The presented elasto-plastic model indicated that permanent waviness/fluting can be generated with elasto-plastic model simulations. Both studied cases presented in this paper, together with earlier studies at [33], proved the usefulness of the model in predicting the challenging deformation phenomena of orthotropic paper sheets.

\section{ACKNOWLEDGEMENTS}

Simulations were performed by a commercial software ABAQUS which was licensed to CSC (the Finnish IT center for science).

\section{REFERENCES}

[1] M. Alava, K. Niskanen, The physics of paper. Reports on Progress in Physics, 69, 669$723,2006$.

[2] L.S. Nordman, Laboratory investigations into the dimensional stability of paper. Tappi, 41, 23-50, 1958.

[3] D.J. Fahey, W.A. Chilson, Mechanical treatments for improving dimensional stability of paper. Tappi, 46, 393-399, 1963. 
[4] L. Salmen, C. Fellers, M. Htun, The development and release of dried-in stresses in paper. Nordic Pulp and Paper Research Journal, 2, 44 48, 1987.

[5] T. Uesaka, D. Qi, Hygroexpansivity of paper: effects of fibre-to-fibre bonding. Journal of Pulp and Paper Science, 20, 175-179, 1994.

[6] Å. Lyne, C. Fellers, P. Kolseth, The effect of filler on hygroexpansivity. Nordic Pulp and Paper Research Journal, 11, 152-163, 1996.

[7] A. de Ruvo, S. Lundberg, S. Martin-Löf, C. Södermark, Influence of temperature and humidity on the elastic and expansional properties of paper and the constituent fibre. The Fundamental Properties of Paper Related to Its Uses, Transactions of the Symposium, London, England, 1976.

[8] V. Setterholm, E.W. Kuenzi, Fiber orientation and degree of restraint during drying - effect of tensile anisotropy of paper handsheet. Tappi, 53, 1915-1920, 1970.

[9] P. Glynn, H.W.H. Jones, W. Gallay, Drying stresses and curl in paper. Pulp and Paper Magazine of Canada, 62, 39-48, 1961.

[10] T. Leppänen, A.-L. Erkkilä, J. Hämäläinen, Effect of fiber orientation structure on simulated cockling of paper. Pulp and Paper Canada, 109, 31-38, 2008.

[11] M.W. Johnson, T.J. Urbanik, A nonlinear theory for elastic plates with application to characterizing paper properties. Journal of Applied Mechanics, 51, 146-152, 1984.

[12] M.W. Johnson, T.J. Urbanik, Buckling of axially loaded, long rectangular paperboard plates. Wood and Fiber Science, 19, 135-146, 1987.

[13] J.C. Suhling, R.E. Rowlands, M.W. Johnson, D.E. Gunderson, Tensorial strength analysis of paperboard. Experimental Mechanics, 25, 75-84, 1985.

[14] C. Fellers, B. Westerlind, A. de Ruvo, An investigation of the biaxial failure envelope of paper - experimental study and theoretical analysis. Proceedings of Fundamental Research Symposium, Cambridge, England, 1983.

[15] J. Castro, M. Ostoja-Starzewski, Elasto-plasticity of paper. International Journal of Plasticity, 19, 2083-2098, 2003.

[16] P. Mäkelä, S. Östlund, Orthotropic elastic-plastic material model for paper materials. International Journal of Solids and Structures, 40, 5599-5620, 2003.

[17] Q.S. Xia, M.C. Boyce, D.M. Parks, A constitutive model for the anisotropic elastic-plastic deformation of paper and paperboard. International Journal of Solids and Structures, 39, 4053-4071, 2002.

[18] J.P. Brezinski, The creep properties of paper. Tappi, 39, 116-128, 1956.

[19] J. Lif, S. Östlund, C. Fellers, Applicability of anisotropic viscoelasticity of paper at small deformations. Mechanics of Time-Dependent Materials, 2, 245-267, 1999.

[20] W. Lu, L.A. Carlsson, Influence of viscoelastic behavior on curl of paper. Mechanics of Time-Dependent Materials, 5, 79-100, 2001. 
[21] M.G. Pecht, M.W. Johnson, R.E. Rowlands, Constitutive equations for the creep of paper. Tappi Journal, 67, 106-108, 1984.

[22] M.G. Pecht, M.W. Johnson, The strain response of paper under various constant regain states. Tappi Journal, 68, 90-93, 1985.

[23] J.L. Rand, A nonlinear viscoelastic creep model. Tappi Journal, 78, 178-182, 1995.

[24] T. Uesaka, K. Murakami, R. Imamura, Two-dimensional linear viscoelasticity of paper. Wood Science and Technology, 14, 131-142, 1980.

[25] T. Uesaka, General formula for hygroexpansion of paper. Journal of Material Science, 29, 2373-2377, 1994.

[26] S. Lavrykov, B.V. Ramarao, Å.L. Lyne, The planar transient hygroexpansion of copy paper: experiments and analysis. Nordic Pulp and Paper Research Journal, 19, 183-190, 2004.

[27] T. Wahlström, K. Adolfsson, S. Östlund, C. Fellers, Numerical modelling of the cross direction shrinkage profile in a drying section: a first approach. Proceedings of the 1999 TAPPI International Paper Physics Conference, San Diego, USA, 1999.

[28] K.C. Yeh, J.M. Considine, J.C. Suhling, The influence of moisture content on the nonlinear constitutive behavior of cellulosic materials. Tappi Proceedings: 1991 International Paper Physics Conference, Havaii, USA, 1991.

[29] N. Banichuk, J. Jeronen, M. Kurki, P. Neittaanmäki, T. Saksa, T. Tuovinen, On the limit velocity and buckling phenomena of axially moving orthotropic membranes and plates. International Journal of Solids and Structures, 48, 2015-2025, 2011.

[30] A. Kulachenko, P. Gradin, H. Koivurova, Modelling the dynamical behaviour of a paper web. Part I. Computers \& structures, 85, 131-147, 2007.

[31] C. Land, T. Wahlström, L. Stolpe, Moisture streaks and their relation to baggy paper webs. Journal of Pulp and Paper Science, 34, 234-239, 2008.

[32] D. Roisum, The mechanics of wrinkling. Tappi Journal, 79, 217-226, 1996.

[33] A.-L. Erkkilä, T. Leppänen, J. Hämäläinen, T. Tuovinen, Hygro-elasto-plastic model for planar orthotropic material. International Journal of Solids and Structures, 62, 66-80, 2015.

[34] J. Pijselman, J. Poustis, Curl of multiply papers - an analytical study. Svensk Papperstidning, 85, 177-184, 1982.

[35] L. Carlsson, C. Fellers, M. Htun, Curl and two-sidedness of paper. Svensk Papperstidning, 83, 194-197, 1980.

[36] F. Bloom, D.W. Coffin, Modelling the hygroscopic buckling of layered paper sheets. Mathematical and Computer Modelling, 31, 43-60, 2000. 
[37] T. Leppänen, J. Sorvari, A.-L. Erkkilä, J. Hämäläinen, Mathematical modelling of moisture induced out-of-plane deformation of a paper sheet. Modelling and Simulation in Materials Science and Engineering, 13, 841-850, 2005.

[38] A. Kulachenko, P. Gradin, T. Uesaka, Tension wrinkling and fluting in heatset web offset printing process - post-buckling analysis. Proceedings of 13th Pulp and Paper Fundamental Research Symposium, Cambridge, England, 2005.

[39] P. Lipponen, T. Leppänen, J. Kouko, J. Hämäläinen, Elasto-plastic approach for paper cockling phenomenon: on the importance of moisture gradient. International Journal of Solids and Structures, 45, 3596-3609, 2008.

[40] T. Uesaka, Dimensional stability and environmental effects on paper properties. Handbook of physical testing of paper, Vol I. New York: Marcel Dekker, 2002.

[41] C.C. Habeger, Tension wrinkling and the fluting of light-weight coated papers in weboffset printing. Journal of Pulp and Paper Science, 19, 214-218, 1993.

[42] J.Y. Hung, Paper fluting - results of TEC studies. TEC Systems, DePere. Wisconsin, USA, 1984.

[43] T. Hirabayashi, S. Fujiwara, T. Fukui, Factors of the fluting of coated paper in web-offset printing. Proceedings of Pan-Pacific and International Printing and Graphic Arts Conference, Montreal, Canada, 1998.

[44] D. Coffin, A buckling analysis corresponding to the fluting of lightweight coated webs. Proceedings of International Paper Physics Conference, Victoria, B.C., Canada, 2003.

[45] J. MacPhee, V. Bellini, B.E. Blom, A.D. Cieri, V. Pinzone, R.S. Potter, The effect of certain variables on fluting in heatset web offset printing. Web Offset Assosiation, Affiliate of Printing Industries of America Inc., USA, 2000.

[46] S. Simmons, B. Blom, C. Dreher, D. Dewildt, D. Coffin, Parametric evaluation of web offset fluting. Proceedings of TAGA's 53rd Annual Technical Conference, San Diego, CA, USA, 2001.

[47] A. Kulachenko, P. Gradin, T. Uesaka, Basic mechanisms of fluting formation and retention in paper. Mechanics of materials, 39, 643-663, 2007.

[48] A.-L. Erkkilä, Mechanism and measurements of the layered orientation structure of paper sheets. Licentiate Thesis, University of Jyväskylä, Jyväskylä, Finland, 1995.

[49] A.-L. Erkkilä, P. Pakarinen and M. Odell, Sheet forming studies using layered orientation analysis. Pulp \& Paper Canada, 99, 81-85, 1998.

[50] P. Lipponen, A.-L. Erkkilä, T. Leppänen and J. Hämäläinen, On the importance of inplane shrinkage and through-thickness moisture gradient during drying on cockling and curling phenomena. Transactions of the 14th Fundamental Research Symposium, Oxford, England, 2009.

[51] C. Hii, Hygroexpansivity of paper. M.Sc. Thesis, Helsinki University of Technology, Espoo, Finland, 2008. 
[52] A.-L. Erkkilä, T. Leppänen, J. Hämäläinen, Empirical plasticity models applied for paper sheets having different anisotropy and dry solids content levels. International Journal of Solids and Structures, 50, 2151-2179, 2013.

[53] A.-L. Erkkilä, T. Leppänen, M. Ora, T. Tuovinen, A. Puurtinen, Hygroexpansivity of anisotropic sheets. Nordic Pulp and Paper Research Journal, 30, 325-334, 2015.

[54] R. Hill, A theory of the yielding and plastic flow of anisotropic metals. Proceedings of the Royal Society of London. Series A, Mathematical and Physical Sciences 191, London, England, 1948.

[55] R.F. Gibson, Principles of composite material mechanics, McGraw-Hill Inc., 1994.

[56] ABAQUS, Abaqus documentation, Dassault Systèmes, Providence, RI, USA, 2013.

[57] M.A. Evans, B.A. LeMaire, An investigation into papers for digital printing, Printing Industry Center, Rochester, NY, USA, 2006.

[58] J.-E. Nordström, S. Lindberg, A. Lundström, Human perception and optical measurement of HSWO waviness. International Printing \& Graphics Arts Conference, Bordeaux, France, 2002.

[59] J. Keränen, O. Timofeev, R. Talja, Simulated combined air-cylinder drying process. Proceedings of 3rd Nordic Drying Conference, Karlstad, Sweden, 2005.

[60] S.G. Harding, D. Wessman, S. Stenström, L. Kenne, Water transport during the drying of cardboard studied by NMR imaging and diffusion techniques. Chemical Engineering Science, 56, 5269-5281, 2001. 